\title{
Velamentous Cord: A Dangerous Case Complicated by a Rural Population
}

\author{
Adam M. Franks, MD' ${ }^{1}$, Carolyn A. Curtis, MD', \\ Shawndra B. Barker, MD'1
}

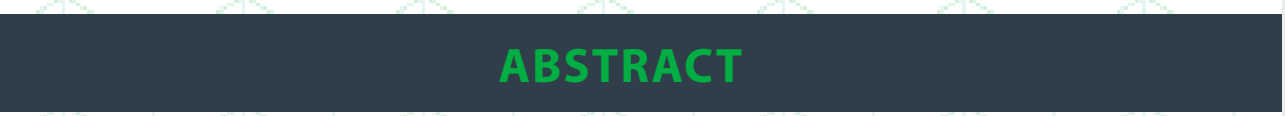

A velamentous cord insertion occurs when the umbilical cord's Wharton jelly fails to reach the placenta, due to early placental atrophy around the insertion site, leaving a segment of unprotected vessels running through the thin membranes of the amniotic sac. This area of weakness exposes a threat to the well-being of the fetus through acute hemorrhage and both acute and chronic restriction of nutrition. With advances in technology, resolution capabilities of ultrasounds allow for antenatal diagnosis, when previously this was impossible. With this knowledge, considerations for screening and management of this pathology are essential when dealing with a rural population that has barriers to accessing health care.
Author affiliations are listed at the end of this article.

Correspondence to: Adam M. Franks, MD Marshall University Joan C. Edwards School of Medicine franks1@marshall.edu

\section{KEYWORDS}

velamentous cord insertion, screening, rural

\section{INTRODUCTION}

Most of West Virginia is classified as rural, ${ }^{1}$ leaving inhabitants of the state less access to state of the art medical care. An American College of Obstetrics and Gynecology (ACOG) Committee Opinion in 2014 found that less than half of rural patients live within 30 minutes to proper medical care and nearly $14 \%$ are outside an hour. ${ }^{2}$ When that care becomes higher risk, needing more frequent monitoring, or even emergent, requiring immediate intervention, this can become deadly.

Abnormal insertion of the umbilical cord occurs in $7.8 \%$ of pregnancies and may consist of marginal cord insertion $(\mathrm{MCl})$, velamentous cord Insertion (VCI) and vasa previa (VP). ${ }^{3}$ When the placenta atrophies around the site of cord insertion early in the gestation, this leaves the umbilical vessels without the Wharton jelly's supportive protection, a $\mathrm{VCl}$ occurs. These exposed vessels are in danger of acute or chronic compression, inhibiting the flow of oxygen and other nutrients to the fetus, resulting in a significantly lower birth weight (3257.0 g vs. 3487.4 $g, \leq 0.001) .{ }^{4}$ They are also at risk for acute vessel rupture, leading to rapid exsanguination of the fetus. The risk of perinatal death at term is tripled for those with a VCl, with survival decreasing from $97 \%$ to $44 \%$ if undiagnosed. 3 . While vessel hemorrhage occurs primarily around the time of rupture of membranes, compression can occur at any time from antepartum to the end of third stage of labor.

Before the advances in ultrasound technology, a placenta's VCl was discovered at the time of placental delivery ... usually incidentally after its successful delivery, but occasionally at the moment of diagnosing a fetal demise either incidentally or from a hemorrhage. As the resolution of ultrasounds improved, it became possible to discover the presence of this and other $\mathrm{MCl}$ pathology as early as the second trimester. ${ }^{6}$ Armed with this knowledge, new clinical screenings and decisions, ones never contemplated previously, must be, due the dangers inherent to an unprotected segment of cord. ${ }^{7}$ Through this case, we will explore the dangers and management of a known $\mathrm{VCl}$ in a rural patient. 


\section{CASE PRESENTATION}

$\mathrm{A} \mathrm{VCl}$, without $\mathrm{VP}$, was noted on a routine screening anatomy ultrasound for TB, a 31 year old gravida 1, para 0 at 20 weeks. Maternal Fetal Medicine (MFM) was consulted to develop a plan for monitoring and delivery. Growth ultrasounds were scheduled every four weeks from viability until delivery and weekly testing with biophysical profiles (BPP) and non-stress testing (NST) at 34 weeks. Due to a forty-five minute distance from medical care, an induction was planned at 39 weeks, prior to membrane rupture. Despite developing pregnancy induced hypertension, testing remained reassuring until 35 week, 4 days, at which time her NST was Category 2 with two one minute decelerations to $70 \mathrm{bpm}$. Concerned for potential cord compromise, the patient was sent to Labor and Delivery for continuous monitoring and ripening with Cervidil followed by augmentation with Pitocin. Throughout the first stage of labor, the patient's fetal heart tracing (FHT) pattern was Category 2 with continued intermittent variable decelerations with an otherwise reassuring pattern. During the second stage of labor, her membranes spontaneously ruptured, causing a five minute period of fetal bradycardia to $90 \mathrm{bpm}$. It was decided prudent to shorten the second stage of labor and deliver the infant with low forceps, delivering a female weighing 2,353 grams with APGARs of $6 \& 7$ at 1 minute and 5 minutes, respectively. The placenta was delivered with gentle cord traction, without cord avulsion. NICU was in attendance but the infant remained in the wellborn nursery, doing well and discharged on the third day of life. Figure 1 depicts the velamentous cord insertion from this case.

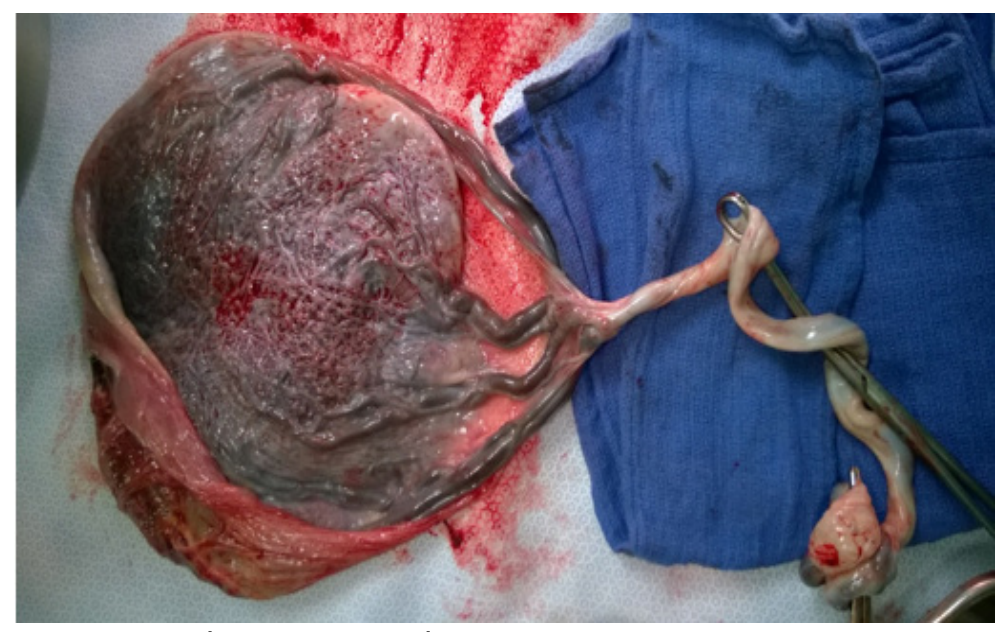

FIGURE 1. Velamentous cord insertion

\section{DISCUSSION}

As early as one week after fertilization, the blastocyst will start to implant on the uterine wall. Where that occurs, determines how successfully the placenta grows. As the trophoblastic cells continue to differentiate, the placenta grows into the organ that will be responsible for transportation of oxygen and nutrients to the fetus and waste back toward the mother. This development of the placenta is completed by $18-20$ weeks but continues to enlarge throughout the remainder of the pregnancy. ${ }^{5}$

If the development of the placenta occurs improperly, a velamentous cord insertion ( $\mathrm{VCl}$ ) can occur. As the vessels course through the membranes, unprotected by the Wharton's jelly, they are vulnerable to both compression and compromise. ${ }^{3,5}$ This compression can cause chronic distress and growth restriction before labor. The compromise can cause catastrophic hemorrhage and exsanguination in labor, starting as early as membrane rupture.

Many of the risk factors seen with $\mathrm{VCl}$ are commonly seen in the WV patient population. Studies show that smoking $(\mathrm{OR}=1.2,95 \% \mathrm{Cl}=1.1-1.3)$, obesity $(\mathrm{OR}=1.83,95 \% \mathrm{Cl}=1.3-2.57)$, asthma $(\mathrm{OR}=1.23$, $95 \% \mathrm{Cl}=1.1-1.3)$, chronic hypertension $(\mathrm{OR}=1.3$, $95 \% \mathrm{Cl}=1.0-1.6)$, type 1 diabetes $(\mathrm{OR}=1.45,95 \% \mathrm{Cl}$ $=1.1-1.9)$ and gestational diabetes $(\mathrm{OR}=1.4,95 \%$ $\mathrm{Cl}=1.2-1.7)$, and pre-eclampsia $(\mathrm{OR}=1.5,95 \% \mathrm{Cl}$ $=1.4-1.7)$ all increase the risk of $\mathrm{VCl} .{ }^{3,4,5}$ All of these conditions are rampant in West Virginia. Screening for VP based on risk factors was found to be cost-

effective. ${ }^{7}$ Screening could be extrapolated to $\mathrm{VCl}$ and easily be accomplished with a 20 week ultrasound.

Current literature gives recommendations for management of VP. ${ }^{6}$ The literature for VCI management, especially in an underserved area, is less defined. If $\mathrm{VCl}$ is present, a plan needs to be developed for potential growth restriction and fetal compromise. Growth ultrasounds every 4 weeks after viability can detect a restriction of nutrients, while twice weekly testing with BPP/NST will monitor fetal well-being. In an underserved area, requiring 
significant travel in the event of an emergency, an elective induction should be initiated prior to labor or rupture of membranes (ROM).

In the first and second stages of labor the fetus is at risk for both compression and compromise injuries. Compression injures of the umbilical cord may occur either spontaneously or by the force of uterine contractions and will present by variable decelerations on the fetal heart tracing on continuous monitoring in labor. Compromise injuries of the umbilical cord are evident by prolonged fetal bradycardia and require emergent delivery. An en caul delivery, where delivery is attempted prior to membrane rupture, should be considered.

Finally, the third stage of labor brings its own risks for a $\mathrm{VCl}$. After the delivery of the infant, gentle traction is placed on the cord to hasten the delivery of the placenta. The lack of Wharton's jelly adding extra strength to exposed membranes and vessels increases the likelihood of avulsion of the cord from the placenta. This necessitates manual, and at times surgical, removal of the placenta increasing the risk of retained products of conception or surgical morbidities. This risk can be lessened with a more conservative management, allowing the placenta to deliver spontaneously.

\section{CONCLUSION}

Though it is a rare condition, the exposed insertion of a velamentous cord can create risk not only for chronic growth restriction from poor transport of nutrition, but also emergent life-threatening hemorrhage. With newer technology, this malformation can be reliably identified in the second trimester and then actively managed, thereby reducing morbidity and mortality in a rural patient population.

\section{AUTHOR AFFILIATIONS}

1. Department of Family Medicine, Marshall University Joan C.Edwards School of Medicine Huntington, WV 25701

\section{REFERENCES}

1. West Virginia Department of Health and Human Resources. Bureau for Public Health. January 2015.

2. Committee Opinion No. 586. Obstetrics \& Gynecology. 2014;123(2, PART 1):384-388.

3. Ebbing C, Kiserud T, Johnsen S, Albrechtsen $S$, Rasmussen S. Prevalence, Risk Factors and Outcomes of Velamentous and Marginal Cord Insertions: A Population-Based Study of 634,741 Pregnancies. PLoS ONE. 2013;8(7):e70380.

4. Raisanen S, Georgiadis L, Harju M, Keski-Nisula L, Heinonen S. Risk factors and adverse pregnancy outcomes among births affected by velamentous umbilical cord insertion: a retrospective population-based register study. Eur J Obstet Gynaecol Reprod Biol. 2012;165(2):231-234.

5. Wiedaseck S, Monchek R. Placental and Cord Insertion Pathologies: Screening, Diagnosis, and Management. J Midwifery Womens Health. 2014;59(3):328-335.

6. Rebarber A, Dolin C, Fox N, Klauser C, Saltzman D, Roman A. Natural History of Vasa Previa Across Gestation Using a Screening Protocol. Journal of Ultrasound in Medicine. 2013;33(1):141-147.

7. Cipriano L, Barth W, Zaric G. The costeffectiveness of targeted or universal screening for vasa praevia at 18-20 weeks of gestation in Ontario. BJOG: An International Journal of Obstetrics \& Gynaecology. 2010;117(9):11081118. 